\title{
新型含 1,2,4-三唑环结构的吡唑肪醚化合物的合成及其生物活性
}

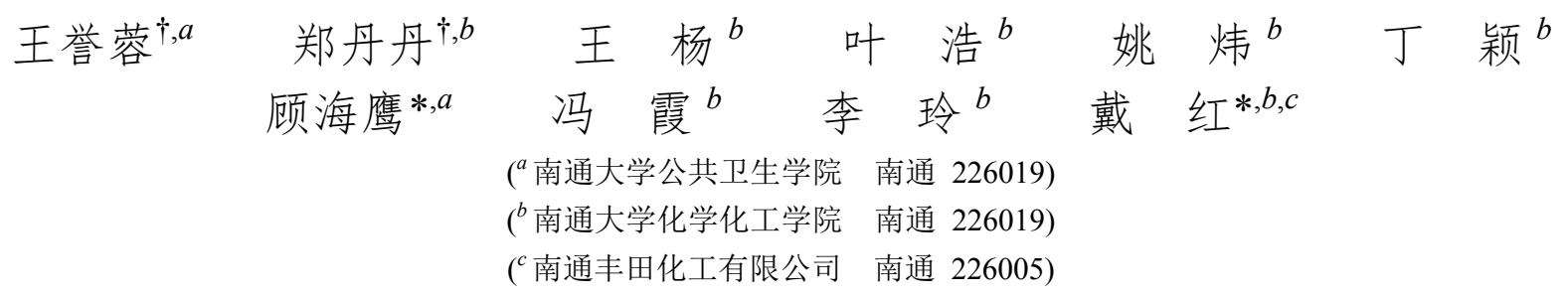

\begin{abstract}
摘要 利用活性亚结构拼接原理, 设计制备了 18 个未见文献报道的新型含 $1,2,4$-三唑环结构单元的吡唑肜醚衍生物. 通过 ${ }^{1} \mathrm{H} N M R 、{ }^{13} \mathrm{C}$ NMR 和元素分析等手段确认了其结构. 初步的生物活性测试结果表明, 部分目标化合物对粘虫、蚜 虫和朱砂叶螨表现出良好的杀灭效果. 在测试浓度为 $500 \mu \mathrm{g} / \mathrm{mL}$ 时，目标化合物对粘虫的致死率在 $90 \% \sim 100 \%$, 与对 照药阿维菌素的杀虫效果相近, 其中有 4 个化合物对蜴虫的致死率均为 100\%, 与对照药吡虫啉的防效相当; 1 -甲基-3甲基-5-(3,5-二氟苯氧基)-1H-吡唑-4-甲醛- $O$-[4-(1H-1,2,4-三唑-1-基)苯甲基]肜(8p)对朱砂叶螨的致死率为 $100 \%$, 与对 照药唑螨酯的防效相当. 当测试浓度降为 $100 \mu \mathrm{g} / \mathrm{mL}$ 时, 5 个化合物对粘虫的致死率在 $90 \% \sim 100 \%, 3$ 个化合物对蚜虫 的致死率在 $80 \% \sim 100 \%$; 化合物 8p 对朱砂叶螨的致死率为 $80 \%$. 当测试浓度降至 $20 \mu \mathrm{g} / \mathrm{mL}$ 时, 2 个化合物对粘虫的致 死率分别为 $75 \%$ 和 $70 \%$. 另外, 部分化合物对人肝癌(SMMC-7721)细胞显示出一定的抗肿瘤活性.
\end{abstract}

关键词 1,2,4-三㭫; 吡唑肜醚; 合成; 生物活性

\section{Synthesis and Bioactivities of Novel Pyrazole Oxime Ethers Containing 1,2,4-Triazole Moiety}

\author{
Wang, Yurong ${ }^{\dagger, a}$ \\ Zheng, Dandan ${ }^{\dagger, b}$ \\ Wang, Yang ${ }^{b}$ \\ Ye, $\mathrm{Hao}^{b}$ \\ Yao, $\mathrm{Wei}^{b}$ \\ Ding, Ying ${ }^{b}$ \\ Gu, Haiying ${ }^{*, a} \quad$ Feng, Xia $^{b} \quad$ Li, Ling $^{b} \quad$ Dai, Hong ${ }^{*, b, c}$ \\ ( ${ }^{a}$ School of Public Health, Nantong University, Nantong 226019) \\ $\left({ }^{b}\right.$ College of Chemistry and Chemical Engineering, Nantong University, Nantong 226019) \\ ( ${ }^{c}$ Nantong Fengtian Chemical Industry Co. Ltd, Nantong 226005)
}

\begin{abstract}
Eighteen novel pyrazole oxime ethers containing 1,2,4-triazole moiety were designed and synthesized according to the method of active substructure combination. Their structures were characterized by ${ }^{1} \mathrm{H}$ NMR, ${ }^{13} \mathrm{C}$ NMR, and elemental analyses. Preliminary bioassay showed that some target compounds showed good insecticidal activities against Oriental armyworm, Aphis medicaginis and Tetranychus cinnabarinus. At the concentration of $500 \mu \mathrm{g} / \mathrm{mL}$, target compounds had insecticidal activity against Oriental armyworm with $90 \% \sim 100 \%$, which were similar to the control of avermectin, four compounds showed $100 \%$ insecticidal activity against Aphis medicaginis, which were similar to imidacloprid. The lethal rate of $1-$ methyl-3-methyl-5-(3,5-difluorophenoxy)-1H-pyrazole-4-carbaldehyde- $O$-[4-(1H-1,2,4-triazol-1-yl)phenylmethyl]oxime (8p) against Tetranychus cinnabarinus was $100 \%$, which was similar to that of fenpyroximate. When the concentration was reduced to $100 \mu \mathrm{g} / \mathrm{mL}$, six compounds exhibited insecticidal activity against Oriental armyworm with $90 \% \sim 100 \%$, three compounds showed insecticidal activity against Aphis medicaginis with $80 \% \sim 100 \%$, and compound $8 \mathbf{p}$ had acaricidal activity against Tetranychus cinnabarinus with $80 \%$. At the concentration of $20 \mu \mathrm{g} / \mathrm{mL}$, the lethal rates of two compounds against Oriental armyworm were $75 \%$ and $70 \%$, respectively. Additionally, some compounds exhibited certain anti-tumor activity against human hepatocellular carcinoma SMMC-7721 cells.
\end{abstract}

Keywords 1,2,4-triazole; pyrazole oxime ether; synthesis; bioactivity

* Corresponding authors. E-mail: guhy99@21cn.com; daihong_2015@aliyun.com

Received February 25, 2019; revised March 22, 2019; published online April 2, 2019.

Project supported by the National Natural Science Foundation of China (Nos. 21874077, 21475070, 21372135), and the Science and Technology Project Fund of Nantong City (No. MS12017022-4).

国家自然科学基金(Nos. 21874077, 21475070,21372135)和南通市科技计划(No. MS12017022-4)资助项目.

$\dagger$ 共同第一作者(These authors contributed equally to this work). 
近年来, 随着大量具有低毒性和较高生物活性的含 氮杂环化合物在文献中被报道, 含氮杂环成为新药创制 研究的热点领域之一 ${ }^{[1 \sim 10]}$. 吡唑肜醚衍生物作为含氮杂 环家族中的重要成员, 因其优异的杀菌、杀虫、抗肿瘤 和抗病毒等生物活性而倍受广大药物化学工作者的关 注 ${ }^{[11 \sim 14]}$. 目前, 具有多种生物活性的吡唑肜醚类化合物 相继被开发出来, 如日本 Nihon Nohyaku 公司成功开发 的杀螨剂唑螨酯(Fenpyroximate, 图 1), 该化合物对多 种植食性螨虫表现出良好的防治效果 ${ }^{[15]}$. 后来药物学 家们纷纷以唑螨酯为药物先导, 衍生制备出了具有多样 生物活性的吡唑肜醚衍生物. Zou 等 ${ }^{[16]}$ 合成的含取代吡 啶环的吡唑肜醚化合物 $\mathbf{A}$ (图 1)呈现出良好杀虫活性. 在测试浓度为 $50 \mu \mathrm{g} / \mathrm{mL}$ 时, 化合物 $\mathbf{A}$ 对蚜虫的致死率 达 $100 \%$. Dai 等 ${ }^{[17]}$ 报道的含取代噻唑环的吡唑朊醚衍生 物 $\mathbf{B}$ (图 1)表现出良好的杀虫与杀螨作用, 在测试浓度 为 $200 \mu \mathrm{g} / \mathrm{mL}$ 时，化合物 B 对小菜蛾的致死率为 $100 \%$, 对螨虫的致死率为 $95 \%$. Shen 等 ${ }^{[18]}$ 合成的含取代噁唑环 的吡唑肜醚衍生物 $\mathbf{C}$ (图 1)具有良好的杀虫、杀螨效果, 在测试浓度为 $500 \mu \mathrm{g} / \mathrm{mL}$ 时, 化合物 $\mathbf{C}$ 对螨虫、粘虫和 蚜虫的致死率分别为 $90 \%, 100 \%$ 和 $100 \%, \mathrm{Lü}$ 等 ${ }^{[19]}$ 报道 的含取代苯环结构的吡唑肟醚化合物 D 对 PI $3 \mathrm{~K} \gamma$ 细胞和 淋巴结细胞显示出良好的抑制效果, 化合物 D 对 PI $3 \mathrm{~K} \gamma$ 细胞和淋巴结细胞的 $\mathrm{IC}_{50}$ 值分别为和 0.28 和 1.18 $\mu \mathrm{mol} \cdot \mathrm{L}^{-1}$, 此外, Dai 等 ${ }^{\left[{ }^{[20]}\right.}$ 合成的含取代噻二唑环结构的 吡唑肜醚衍生物 $\mathbf{E}$ (图 1)对人肝癌细胞 Huh-7 和人肤腺 癌细胞 Panc-1 具有良好的抗肿瘤效果, 其 $\mathrm{IC}_{50}$ 值分别为 10.17 和 $12.22 \mu \mathrm{mol} \cdot \mathrm{L}^{-1}$. 另外, $1,2,4$-三唑环是含三个氮 原子的五元芳香杂环，1,2,4-三唑类化合物具有优良的 生物活性，已在农药和医药领域得到了广泛的应用，如 杀虫 ${ }^{[21]}$ 、杀菌 ${ }^{[22]} 、$ 除草 ${ }^{[23]} 、$ 抗惊厥 ${ }^{[24]} 、$ 抗癌 ${ }^{[25]}$ 等活性. 最近 Jia 等 ${ }^{[26]}$ 报道的含氨基甲酸酯单元的 1,2,4-三唑类 化合物 F (图 1)对蚜虫表现出较好的杀虫效果, 在测试 浓度为 $500 \mu \mathrm{g} / \mathrm{mL}$ 时，化合物 $\mathbf{F}$ 对蚜虫的致死率大于 $90 \%$. Wei 等 ${ }^{[27]}$ 合成的含三氮烯骨架结构的 1,2,4-三唑衍 生物 $\mathbf{G}$ (图 1)对人膀胱癌细胞 J82 和人前列腺癌细胞 DU145 呈现出良好的抗肿瘤作用，其 $\mathrm{IC}_{50}$ 值分别为 5.512 和 $13.69 \mu \mathrm{mol} \cdot \mathrm{L}^{-1}$. 鉴于此, 为了寻找具有较高生 物活性的新型吡唑肜醚类衍生物, 本文以唑螨酯为先导 化合物，采用活性亚结构拼接的方法，将取代 1,2,4-三 唑结构单元引入到吡唑肜醚分子结构中，设计合成了一 系列新型含 1,2,4-三唑环结构的吡唑肟醚衍生物(图 2). 同时对所制备的目标化合物进行了初步的生物活性测 试. 结果显示部分目标物具有较好的杀虫活性，部分目 标物还表现出良好的抗肿瘤作用. 目标化合物的合成路 线如 Scheme 1 所示.

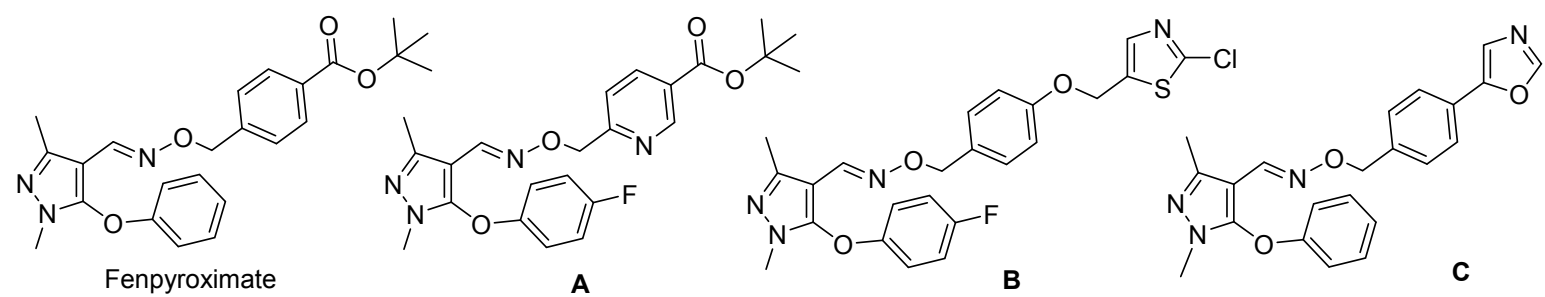<smiles>Fc1ccccc1CON=Cc1cn(-c2ccccc2)nc1-c1ccccc1</smiles><smiles>Cc1nnsc1CON=Cc1c(C)nn(C)c1Oc1cccc(F)c1F</smiles><smiles>CCOC(=O)Nc1nc(-c2ccc(F)cc2)nn1CC</smiles><smiles>CN(C)N=Nc1ccc(-c2nnc(SCc3ccc(Cl)cc3Cl)n2N)cc1</smiles>

图 1 唑螨酯和化合物 $\mathbf{A} \sim \mathbf{G}$ 的化学结构式

Figure 1 Chemical structures of fenpyroximate and compounds $\mathbf{A} \sim \mathbf{G}$
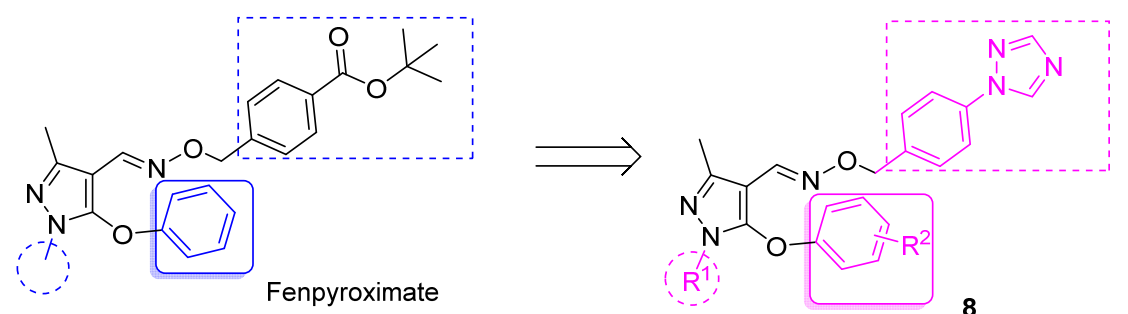

图 2 目标化合物 $\mathbf{8}$ 的分子设计示意图

Figure 2 Design strategy of the title compounds 8 

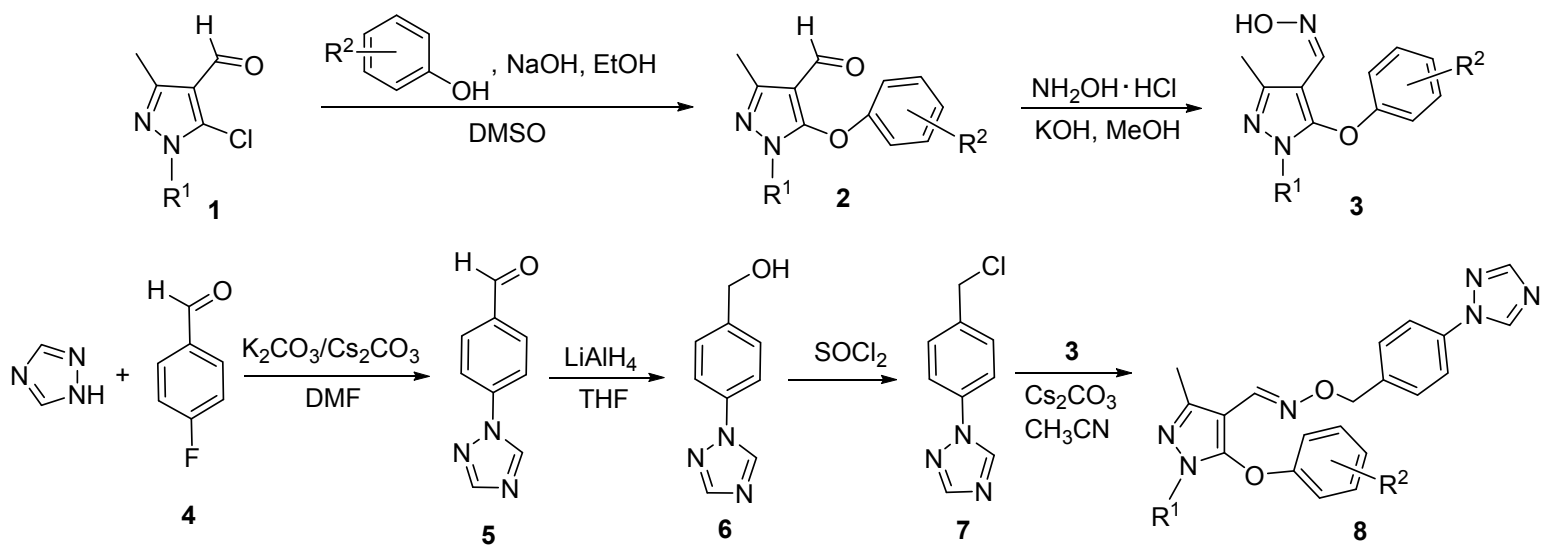

8a: $R^{1}=\mathrm{CH}_{3}, \mathrm{R}^{2}=2-\mathrm{OCH}_{3} ; \mathbf{8 b}: \mathrm{R}^{1}=\mathrm{CH}_{3}, \mathrm{R}^{2}=4-\mathrm{OCH}_{3} ; \mathbf{8 c}: \mathrm{R}^{1}=\mathrm{CH}_{3}, \mathrm{R}^{2}=4-\mathrm{OCF}_{3} ; \mathbf{8 d}: \mathrm{R}^{1}=\mathrm{CH}_{3}, \mathrm{R}^{2}=3-\mathrm{CH}_{3} ; \mathbf{8 e}: \mathrm{R}^{1}=\mathrm{CH}_{3}, \mathrm{R}^{2}=4-\mathrm{CH}_{3} ;$ 8f: $R^{1}=\mathrm{CH}_{3}, \mathrm{R}^{2}=\mathrm{H} ; \mathbf{8 g}: \mathrm{R}^{1}=\mathrm{CH}_{3}, \mathrm{R}^{2}=2-\mathrm{F} ; \mathbf{8} \mathbf{h}: \mathrm{R}^{1}=\mathrm{CH}_{3}, \mathrm{R}^{2}=3-\mathrm{F} ; \mathbf{8 i}: \mathrm{R}^{1}=\mathrm{CH}_{3}, \mathrm{R}^{2}=4-\mathrm{F} ; \mathbf{8 j}: \mathrm{R}^{1}=\mathrm{CH}_{3}, \mathrm{R}^{2}=4-\mathrm{Cl} ; \mathbf{8 k}: \mathrm{R}^{1}=\mathrm{CH}_{3}, \mathrm{R}^{2}=2-$ $\mathrm{Br}$; 8I: $\mathrm{R}^{1}=\mathrm{CH}_{3}, \mathrm{R}^{2}=3-\mathrm{Br} ; \mathbf{8 m}: \mathrm{R}^{1}=\mathrm{CH}_{3}, \mathrm{R}^{2}=4-\mathrm{Br} ; \mathbf{8 n}: \mathrm{R}^{1}=\mathrm{CH}_{3}, \mathrm{R}^{2}=2,3-\mathrm{F}_{2} ; \mathbf{8 o}: \mathrm{R}^{1}=\mathrm{CH}_{3}, \mathrm{R}^{2}=2,4-\mathrm{F}_{2} ; \mathbf{8 p}: \mathrm{R}^{1}=\mathrm{CH}_{3}, \mathrm{R}^{2}=3,5-\mathrm{F}_{2} ; \mathbf{8 q}:$ $\mathrm{R}^{1}=\mathrm{CH}_{3}, \mathrm{R}^{2}=2,4-\mathrm{Cl}_{2} ; 8 \mathrm{r}: \mathrm{R}^{1}=4-\mathrm{CH}_{3} \mathrm{C}_{6} \mathrm{H}_{4}, \mathrm{R}^{2}=4-\mathrm{CH}_{3}$

图式 1 目标化合物 8 的合成路线

Scheme 1 Synthetic route of the target compounds 8

\section{1 结果与讨论}

\section{1 目标化合物的合成}

我们以目标化合物 $\mathbf{8 b}$ 为研究对象, 探索了不同的 反应条件对化合物 $8 \mathrm{~b}$ 单步收率的影响. 由表 1 可以看 出, 选用碳酸铯作缚酸剂, 乙腈作溶剂, 加热回流反应 $12 \mathrm{~h}$, 是合成目标物的较佳方法, 化合物 $\mathbf{8 b}$ 的单步收率 达到 $72 \%$. 通过该方法顺利地合成了其它目标化合物, 通过 ${ }^{1} \mathrm{H}$ NMR、 ${ }^{13} \mathrm{C}$ NMR 和元素分析等手段对其结构进 行了确证.

表 1 不同反应条件对目标化合物 $8 \mathrm{~b}$ 合成收率的影响 Table 1 Effects of reaction conditions on the synthesis of the title compound $\mathbf{8 b}$

\begin{tabular}{cllcc}
\hline Entry & \multicolumn{1}{c}{ Base } & \multicolumn{1}{c}{ Solvent } & Temp. $/{ }^{\circ} \mathrm{C}$ & Yield/\% \\
\hline 1 & $\mathrm{Et}_{3} \mathrm{~N}$ & $\mathrm{CH}_{3} \mathrm{COCH}_{3}$ & Reflux & 0 \\
2 & $\mathrm{Pyridine}_{3}$ & $\mathrm{CH}_{3} \mathrm{COCH}_{3}$ & Reflux & 0 \\
3 & $\mathrm{NaHCO}_{3}$ & $\mathrm{CH}_{3} \mathrm{COCH}_{3}$ & Reflux & 0 \\
4 & $\mathrm{~K}_{2} \mathrm{CO}_{3}$ & $\mathrm{CH}_{3} \mathrm{COCH}$ & Reflux & 29 \\
5 & $\mathrm{NaHCO}_{3}$ & $\mathrm{CH}_{3} \mathrm{CN}$ & Reflux & 0 \\
6 & $\mathrm{~K}_{2} \mathrm{CO}_{3}$ & $\mathrm{CH}_{3} \mathrm{CN}$ & Reflux & 61 \\
7 & $\mathrm{Cs}_{2} \mathrm{CO}_{3}$ & $\mathrm{CH}_{3} \mathrm{CN}$ & Reflux & 72 \\
8 & $\mathrm{NaHCO}_{3}$ & $\mathrm{DMF}$ & 90 & 0 \\
9 & $\mathrm{~K}_{2} \mathrm{CO}_{3}$ & $\mathrm{DMF}$ & 90 & 50 \\
10 & $\mathrm{Cs}_{2} \mathrm{CO}_{3}$ & DMF & 90 & 65 \\
\hline
\end{tabular}

\section{2 化合物的图谱解析}

以目标化合物 8a 的核磁数据为例进行解析. $\delta 8.55$ 处的单峰为三唑环上一个氢的吸收峰; $\delta 8.11$ 处的单峰 为三坐环上另外一个氢的吸收峰; $\delta 7.78$ 处的单峰对应 于 $\mathrm{CH}=\mathrm{N}$ 氢的吸收峰; $\delta 7.62$ 处的双重峰为与三唑环相 连的苯环上两个氢的吸收峰; $\delta 7.42$ 处的双重峰为与三 唑环相连的苯环上另外两个氢的吸收峰; $\delta$ 6.68 7.11
间的多重峰为与氧相连的苯环上四个氢的吸收峰; $\delta$ 5.03 处的单峰对应于和苯环相连的 $\mathrm{CH}_{2}$ 两个氢的吸收 峰; $\delta 3.90$ 处的单峰为苯环 2-位甲氧基上三个氢的吸收 峰; $\delta 3.63$ 处的单峰为吡唑环 1-位 $N$-甲基上三个氢的吸 收峰; $\delta 2.35$ 处的单峰为吡唑环 3-位甲基上三个氢的吸 收峰; $\delta 75.0$ 处的峰为和苯环相连的 $\mathrm{CH}_{2}$ 碳原子的信号 峰; $\delta 56.1$ 处的峰为苯环 2-位甲氧基碳原子的信号峰; $\delta$ 34.2 处的峰为吡唑环 1-位 $N$-甲基碳原子的信号峰; $\delta$ 14.9 处的峰为吡唑环 3-位甲基碳原子的信号峰.

\section{3 化合物的杀虫活性}

目标化合物 8a $\sim 8 \mathbf{r}$ 对粘虫( Oriental armyworm)、蚜 虫 (Aphis medicaginis) 和朱砂叶螨 (Tetranychus cinnabarinus) 的杀虫活性测试结果见表 2. 初步的生物活性 测试结果显示, 所有目标化合物对粘虫均表现出较好的 杀虫活性，但无明显的规律. 在测试浓度为 $500 \mu \mathrm{g} / \mathrm{mL}$ 时，化合物 8a $\sim 8$ 对粘虫具有 $90 \%$ 以上致死率，与对照 药阿维菌素的杀虫效果相当，当 $\mathrm{R}^{1}=\mathrm{CH}_{3}$ 时，4-甲氧基 取代物 8b、4-三氟甲氧基取代物 8c、4-甲基取代物 8e、 氢取代物 8f、3-氟取代物 8h、4-溴取代物 8m 和 3,5-二 氟取代物 8p 在测试浓度降为 $100 \mu \mathrm{g} / \mathrm{mL}$ 时，对粘虫仍呈 现出较好的杀虫作用，其致死率分别为 $90 \%, 100 \%$, $60 \%, 90 \%, 70 \%, 100 \%$ 和 $100 \%$. 其中化合物 8b、8c、8f、 $8 \mathrm{~m}$ 和 $8 \mathrm{p}$ 对粘虫的杀灭效果与对照药阿维菌素相近. 当 测试浓度减至 $20 \mu \mathrm{g} / \mathrm{mL}$ 时, 某些化合物对粘虫仍具一 定的杀虫效果，其中当 $\mathrm{R}^{1}=\mathrm{CH}_{3}$ 时，4-三氟甲氧基取代 物 8c 和 3,5-二氟取代物 8p 对粘虫的致死率分别为 75\% 和 $70 \%$. 在测试浓度为 $500 \mu \mathrm{g} / \mathrm{mL}$ 时，部分目标化合物 对蚜虫表现出良好的杀虫效果，其中当 $\mathrm{R}^{1}=\mathrm{CH}_{3}$ 时，4- 
三氟甲氧基取代物 8c、4-甲基取代物 8e、2-氟取代物 8g、 4-溴取代物 $8 \mathrm{~m}$ 和 3,5-二氟取代物 $8 \mathrm{p}$ 对蚜虫的杀灭效果 相对较好，其致死率分别为 $100 \%, 100 \%, 60 \%, 100 \%$ 和 $100 \%$. 其中化合物 $8 \mathrm{c} 、 8 \mathrm{e} 、 8 \mathrm{~m}$ 和 $8 \mathrm{p}$ 对蚜虫的防治效 果与对照药吡虫啉相当. 当测试浓度降到 $100 \mu \mathrm{g} / \mathrm{mL}$ 时, 部分目标化合物对蚜虫仍具有良好的杀虫活性, 其中当 $\mathrm{R}^{1}=\mathrm{CH}_{3}$ 时, 4-甲基取代物 8e、4-溴取代物 8m 和 3,5-二 氟取代物 8p 对蚜虫的杀虫作用相对较好, 其致死率分 别为 $100 \%, 80 \%$ 和 $100 \%$. 另外, 在测试浓度为 500 $\mu \mathrm{g} / \mathrm{mL}$ 时, 某些目标化合物对朱砂叶螨也表现出杀灭作 用. 其中当 $\mathrm{R}^{1}=\mathrm{CH}_{3}$ 时, 3,5-二氟取代物 8p 对朱砂叶螨 的致死率达 $100 \%$, 与对照药唑螨酯的防效接近. 当测 试浓度降为 $100 \mu \mathrm{g} / \mathrm{mL}$ 时, 化合物 $\mathbf{8 p}$ 对朱砂叶螨仍有 $80 \%$ 的防治效果. 整体来看, 化合物 $8 \mathrm{p}\left(\mathrm{R}^{1}=\mathrm{CH}_{3}, \mathrm{R}^{2}=\right.$ $\left.3,5-F_{2}\right)$ 显示出较为广谱的杀虫活性. 这为今后进一步进 行吡唑肜醚类衍生物的结构优化与生物活性研究提供 了重要的试验数据.

\section{4 化合物的抗肿瘤活性}

目标化合物 $8 \mathbf{a} \sim 8 \mathbf{r}$ 对人肝癌 $(S M M C-7721)$ 和人胰 腺癌(Panc-1)细胞株的体外抗肿瘤活性结果如表 3 所示. 从表 3 可以看出, 目标化合物 $8 \mathbf{a} \sim 8 \mathbf{r}$ 对人胰腺癌 Panc-1 细胞均未显示出明显的抑制效果, 部分化合物对人肝癌 SMMC-7721 细胞具有较好的抑制作用, 但无明显的规
律. 目标化合物 $8 d 、 8 f 、 8 k 、 8 p$ 和 $8 r$ 对人肝癌 SMMC7721 细胞的抑制效果相对较好, 其 $\mathrm{IC}_{50}$ 值分别为 14.3 , 15.6, 12.1, 11.8 和 $9.4 \mu \mathrm{mol} / \mathrm{L}$, 优于对照药 5-Fluorouracil 的活性.

\section{2 结论}

本文利用活性基团拼接原理，设计合成了 18 个新 型含取代 1,2,4-三唑结构的吡唑肟醚化合物. 测试结果 表明, 部分化合物呈现出良好的杀虫活性. 在测试浓度 为 $500 \mu \mathrm{g} / \mathrm{mL}$ 时，化合物 $8 \mathbf{a} \sim 8 \mathbf{r}$ 对粘虫的致死率在 $90 \% \sim 100 \%$ ，当测试浓度降至 $100 \mu \mathrm{g} / \mathrm{mL}$ 时，化合物 8b、8c、8f、8m 和 $8 p$ 对粘虫的杀灭效果仍在 $90 \% \sim 100 \%$; 当测试浓度降至 $20 \mu \mathrm{g} / \mathrm{mL}$ 时, 化合物 $8 \mathrm{c}$ 和 $8 \mathrm{p}$ 对粘虫还 具有较好的杀虫效果，其致死率分别为 75\%和 70\%; 化 合物 8e、8m 和 $8 p$ 在测试浓度为 500 和 $100 \mu \mathrm{g} / \mathrm{mL}$ 时对 蚜虫均表现出优良的杀虫作用; 化合物 $\mathbf{8 p}$ 在测试浓度 为 500 和 $100 \mu \mathrm{g} / \mathrm{mL}$ 时对朱砂叶螨显示出较好的杀灭效 果. 此外, 化合物 8d、8f、8k、8p 和 8r 对 SMMC-7721 细胞的抑制作用相对较好, $\mathrm{IC}_{50}$ 值分别为 $14.3,15.6$, $12.1,11.8$ 和 $9.4 \mu \mathrm{mol} / \mathrm{L}$, 高于对照药 5-Fluorouracil 的抑 制活性. 该类化合物的结构衍生与生物活性研究正在进 行中.

表 2 目标化合物 $8 \mathrm{a} \sim 8 \mathrm{r}$ 的活体杀虫活性(死亡率/\%) ${ }^{a}$

Table 2 The in vivo insecticidal activities (mortality/\%) of tile compounds $8 \mathbf{a} \sim \mathbf{8 r}$

\begin{tabular}{|c|c|c|c|c|c|c|c|c|c|c|}
\hline \multirow{2}{*}{ Compd. } & \multicolumn{4}{|c|}{ Oriental armyworm } & \multicolumn{3}{|c|}{ Aphis medicaginis } & \multicolumn{3}{|c|}{ Tetranychus cinnabarinus } \\
\hline & $500 \mu \mathrm{g} / \mathrm{mL}$ & $100 \mu \mathrm{g} / \mathrm{mL}$ & $20 \mu \mathrm{g} / \mathrm{mL}$ & $4 \mu \mathrm{g} / \mathrm{mL}$ & $500 \mu \mathrm{g} / \mathrm{mL}$ & $100 \mu \mathrm{g} / \mathrm{mL}$ & $20 \mu \mathrm{g} / \mathrm{mL}$ & $500 \mu \mathrm{g} / \mathrm{mL}$ & $100 \mu \mathrm{g} / \mathrm{mL}$ & $20 \mu \mathrm{g} / \mathrm{mL}$ \\
\hline $8 a$ & 100 & 0 & - & - & 0 & - & - & 30 & 0 & - \\
\hline $8 b$ & 100 & 90 & 0 & - & 0 & - & - & 0 & - & - \\
\hline $8 c$ & 100 & 100 & 75 & 0 & 100 & 30 & - & 0 & - & - \\
\hline 8d & 100 & 0 & - & - & 0 & - & - & 0 & - & - \\
\hline $8 e$ & 100 & 60 & 0 & - & 100 & 100 & 30 & 0 & - & - \\
\hline $8 f$ & 100 & 90 & 0 & - & 0 & - & - & 0 & - & - \\
\hline $8 g$ & 100 & 0 & - & - & 60 & 0 & - & 0 & - & - \\
\hline $8 \mathrm{~h}$ & 100 & 70 & 0 & - & 0 & - & - & 0 & - & - \\
\hline $8 \mathbf{i}$ & 100 & 0 & - & - & 0 & - & - & 0 & - & - \\
\hline $8 \mathbf{j}$ & 100 & 0 & - & - & 0 & - & - & 0 & - & - \\
\hline $8 k$ & 100 & 0 & - & - & 0 & - & - & 0 & - & - \\
\hline 81 & 100 & 0 & - & - & 0 & - & - & 0 & - & - \\
\hline $8 m$ & 100 & 100 & 0 & - & 100 & 80 & 0 & 40 & 0 & - \\
\hline $8 n$ & 90 & 0 & - & - & 0 & - & - & 0 & - & - \\
\hline 80 & 100 & 0 & - & - & 0 & - & - & 0 & - & - \\
\hline $8 p$ & 100 & 100 & 70 & 0 & 100 & 100 & 0 & 100 & 80 & 0 \\
\hline $8 q$ & 90 & 0 & - & - & 0 & - & - & 0 & - & - \\
\hline $8 r$ & 100 & 0 & - & - & 0 & - & - & 0 & - & - \\
\hline Avermectin & 100 & 100 & 100 & 100 & - & - & - & - & - & - \\
\hline Imidacloprid & - & - & - & - & 100 & 100 & 100 & - & - & - \\
\hline Fenpyroximate & - & - & - & - & - & - & - & 100 & 100 & 100 \\
\hline
\end{tabular}

a- refers to "not tested". 
表 3 目标化合物 8a $~ 8 \mathrm{r}$ 的体外抗肿瘤活性 Table 3 The in vitro cytotoxicity of title compounds $\mathbf{8 a} \sim \mathbf{8 r}$

\begin{tabular}{ccc}
\hline \multirow{2}{*}{ Compd. } & \multicolumn{2}{c}{$\mathrm{IC}_{50} /\left(\mu \mathrm{mol} \cdot \mathrm{L}^{-1}\right)$} \\
\cline { 2 - 3 } $\mathbf{8 a}$ & $\mathrm{S} M \mathrm{C}-7721$ & Panc- 1 \\
$\mathbf{8 b}$ & $>25$ & $>25$ \\
$\mathbf{8 c}$ & $>25$ & $>25$ \\
$\mathbf{8 d}$ & 14.3 & $>25$ \\
$\mathbf{8 e}$ & $>25$ & $>25$ \\
$\mathbf{8 f}$ & 15.6 & $>25$ \\
$\mathbf{8 g}$ & $>25$ & $>25$ \\
$\mathbf{8 h}$ & $>25$ & $>25$ \\
$\mathbf{8 i}$ & $>25$ & $>25$ \\
$\mathbf{8 j}$ & $>25$ & $>25$ \\
$\mathbf{8 k}$ & 12.1 & $>25$ \\
$\mathbf{8 1}$ & 18.5 & $>25$ \\
$\mathbf{8 m}$ & 19.8 & $>25$ \\
$\mathbf{8 n}$ & 17.9 & $>25$ \\
$\mathbf{8 0}$ & $>25$ & $>25$ \\
$\mathbf{8 p}$ & 11.8 & $>25$ \\
$\mathbf{8 q}$ & $>25$ & $>25$ \\
$\mathbf{8 r}$ & 9.4 & $>25$ \\
5 -Fluorouracil & 37.8 & -25 \\
Sorafenib & - & 11.5 \\
\hline & &
\end{tabular}

\section{3 实验部分}

\section{1 仪器与试剂}

X-4 型数字显示熔点测定仪 (北京泰克仪器有限公 司), 温度计未经校正; Yanaco-CHN CORDER MT-3 自 动元素分析仪; Bruker AM-400 型核磁共振仪, 以 $\mathrm{CDCl}_{3}$ 为溶剂, TMS 为内标; 柱层析硅胶为 $\mathrm{H}$ 型(青岛海洋化工 厂, 200 300 目).所用试剂均为分析纯.

\section{2 中间体的制备}

中间体 1 按照文献[28]方法制备, 中间体 $\mathbf{2}$ 和 3 按 照文献[29]方法制备，中间体 5 按照文献[30]方法制备.

\section{$3.34-(1 H-1,2,4-$ 三唑-1-基)苯甲醇(6)的合成}

在一 $100 \mathrm{~mL}$ 圆底烧瓶中, 加入 $10 \mathrm{mmol}$ 中间体 $\mathbf{5}$ 及 $35 \mathrm{~mL}$ 无水四氢呋喃. 冰浴摚拌下，向其分批加入 30 $\mathrm{mmol}$ 四氢铝锂, 加毕, 继续冰浴搅拌 $4 \mathrm{~h}$, 停止反应.向 其反应液中加入适量水，抽滤，滤液用乙酸乙酯 $(50 \times 3$ $\mathrm{mL}$ )萃取. 合并有机相, 无水硫酸钠干燥, 抽滤, 将滤液 浓缩, 所得残余物通过柱层析 $[V$ (石油醚) : $V($ 乙酸乙 酯 $)=4: 1$ ] 分离得中间体 6, 产率 $83 \%$. m.p. 113 $115{ }^{\circ} \mathrm{C} ;{ }^{1} \mathrm{H}$ NMR $\left(400 \mathrm{MHz}, \mathrm{CDCl}_{3}\right) \delta: 8.52(\mathrm{~s}, 1 \mathrm{H}$, Triazole-H), 8.08 (s, $1 \mathrm{H}$, Triazole-H), 7.64 (d, $J=8.0 \mathrm{~Hz}$, $2 \mathrm{H}, \mathrm{ArH}), 7.50$ (d, $J=8.0 \mathrm{~Hz}, 2 \mathrm{H}, \mathrm{ArH}), 4.76\left(\mathrm{~s}, 2 \mathrm{H}, \mathrm{CH}_{2}\right)$; ${ }^{13} \mathrm{C}$ NMR $\left(100 \mathrm{MHz}, \mathrm{CDCl}_{3}\right) \delta: 152.5,141.4,140.8,136.1$, 128.1, 120.1, 64.2. Anal. calcd for $\mathrm{C}_{9} \mathrm{H}_{9} \mathrm{~N}_{3} \mathrm{O}: \mathrm{C} 61.70, \mathrm{H}$ 5.18, N 23.99; found C 61.87, H 5.02, N 23.85.

\subsection{4-(1H-1,2,4-三唑-1-基)苯甲基氯(7)的合成}

在一 $100 \mathrm{~mL}$ 圆底烧瓶中, 加入 $8 \mathrm{mmol}$ 中间体 6 及 $50 \mathrm{~mL}$ 二氯甲烷，冰浴搅拌下，向其中滴加 $50 \mathrm{mmol}$ 氯 化亚砜, 滴毕, 继续冰浴搅拌 $10 \mathrm{~min}$, 然后升温回流 $2 \mathrm{~h}$, 停止反应, 冷却至室温. 向其反应液中加入适量水, 分 层, 有机层依次用饱和碳酸氢钠溶液、饱和氯化钠溶液 洗涤、无水硫酸钠干燥，抽滤、旋蒸除去溶剂，所得残 余物通过柱层析 $[V$ (石油醚 $): V($ 乙酸乙酯 $)=3: 1]$ 分离 得中间体 7, 产率 79\%. m.p. 88 90 ${ }^{\circ} \mathrm{C}$; ${ }^{1} \mathrm{H}$ NMR (400 $\left.\mathrm{MHz}, \mathrm{CDCl}_{3}\right) \delta: 8.62(\mathrm{~s}, 1 \mathrm{H}$, Triazole-H), $8.13(\mathrm{~s}, 1 \mathrm{H}$, Triazole-H), 7.70 (d, $J=8.4 \mathrm{~Hz}, 2 \mathrm{H}, \mathrm{ArH}), 7.55$ (d, $J=8.4$ $\mathrm{Hz}, 2 \mathrm{H}, \mathrm{ArH}), 4.64\left(\mathrm{~s}, 2 \mathrm{H}, \mathrm{CH}_{2}\right) ;{ }^{13} \mathrm{C}$ NMR $(100 \mathrm{MHz}$, $\left.\mathrm{CDCl}_{3}\right) \delta: 152.7,140.9,137.6,136.8,130.1,120.2,45.2$. Anal. calcd for $\mathrm{C}_{9} \mathrm{H}_{8} \mathrm{ClN}_{3}$ : C 55.83, H 4.16, N 21.70; found C 55.70, H 4.01, N 21.81.

\section{5 目标化合物 8 的合成}

在一 $50 \mathrm{~mL}$ 圆底烧瓶中, 加入 $2 \mathrm{mmol}$ 肜中间体 3 、 $5 \mathrm{mmol}$ 碳酸铯及 $20 \mathrm{~mL}$ 无水乙腈，室温下搅拌，向其中 加入 $2.4 \mathrm{mmol}$ 中间体 7. 加毕, 升温回流反应 $10 \sim 22 \mathrm{~h}$. 冷却, 减压过滤, 将滤液浓缩, 所得残余物通过柱层析 $[V$ (石油醚) $: V($ 乙酸乙酯 $)=20: 1]$ 分离得目标化合物 $8 \mathbf{a} \sim 8 \mathbf{r}$.

1-甲基-3-甲基-5-(2-甲氧基苯氧基)- $1 H$-吡唑-4-甲 醛-O-[4-(1H-1,2,4-三唑-1-基)苯甲基]肜(8a)：白色固体, 产率 63\%. m.p. $139 \sim 141{ }^{\circ} \mathrm{C} ;{ }^{1} \mathrm{H}$ NMR $(400 \mathrm{MHz}$, $\left.\mathrm{CDCl}_{3}\right) \delta: 8.55(\mathrm{~s}, 1 \mathrm{H}$, Triazole-H), $8.11(\mathrm{~s}, 1 \mathrm{H}$, Triazole-H), $7.78(\mathrm{~s}, 1 \mathrm{H}, \mathrm{CH}=\mathrm{N}), 7.62(\mathrm{~d}, J=8.8 \mathrm{~Hz}, 2 \mathrm{H}$, $\operatorname{ArH}), 7.42(\mathrm{~d}, J=8.0 \mathrm{~Hz}, 2 \mathrm{H}, \operatorname{ArH}), 6.68 \sim 7.11(\mathrm{~m}, 4 \mathrm{H}$, $\mathrm{ArH}), 5.03\left(\mathrm{~s}, 2 \mathrm{H}, \mathrm{CH}_{2}\right), 3.90\left(\mathrm{~s}, 3 \mathrm{H}, \mathrm{OCH}_{3}\right), 3.63(\mathrm{~s}, 3 \mathrm{H}$, $\left.\mathrm{N}-\mathrm{CH}_{3}\right), 2.35$ (s, 3H, $\left.\mathrm{CH}_{3}\right) ;{ }^{13} \mathrm{C} \mathrm{NMR}\left(100 \mathrm{MHz}, \mathrm{CDCl}_{3}\right.$ ) $\delta: 152.6,148.9,148.4,146.8,145.8,141.2,138.2,136.4$, 129.8, 129.3, 124.6, 120.9, 119.9, 115.8, 112.7, 99.6, 75.0, 56.1, 34.2, 14.9. Anal. calcd for $\mathrm{C}_{22} \mathrm{H}_{22} \mathrm{~N}_{6} \mathrm{O}_{3}: \mathrm{C} 63.15, \mathrm{H}$ 5.30, N 20.08; found C 63.01, H 5.43, N 20.26.

1-甲基-3-甲基-5-(4-甲氧基苯氧基)- $1 \mathrm{H}$-吡唑-4-甲 醛- $O$-[4-(1H-1,2,4-三唑-1-基)苯甲基]肜(8b)：白色固体, 产率 72\%. m.p. 88 90 ${ }^{\circ} \mathrm{C} ;{ }^{1} \mathrm{H}$ NMR $\left(400 \mathrm{MHz}, \mathrm{CDCl}_{3}\right)$ $\delta: 8.56(\mathrm{~s}, 1 \mathrm{H}$, Triazole-H), 8.11 (s, 1H, Triazole-H), 7.80 $(\mathrm{s}, 1 \mathrm{H}, \mathrm{CH}=\mathrm{N}), 7.62(\mathrm{~d}, J=8.8 \mathrm{~Hz}, 2 \mathrm{H}, \operatorname{ArH}), 7.42(\mathrm{~d}$, $J=8.4 \mathrm{~Hz}, 2 \mathrm{H}, \mathrm{ArH}), 6.82$ (s, 4H, ArH), 5.05 (s, 2H, $\mathrm{CH}_{2}$ ), $3.76\left(\mathrm{~s}, 3 \mathrm{H}, \mathrm{OCH}_{3}\right), 3.61\left(\mathrm{~s}, 3 \mathrm{H}, \mathrm{N}-\mathrm{CH}_{3}\right), 2.35(\mathrm{~s}, 3 \mathrm{H}$, $\left.\mathrm{CH}_{3}\right) ;{ }^{13} \mathrm{C}$ NMR $\left(100 \mathrm{MHz}, \mathrm{CDCl}_{3}\right) \delta: 155.8,150.6,148.4$, $146.8,141.2,138.2,137.8,136.4,129.8,119.9,116.3$, $114.9,114.8,99.8,75.0,55.7,34.2,14.7$. Anal. calcd for $\mathrm{C}_{22} \mathrm{H}_{22} \mathrm{~N}_{6} \mathrm{O}_{3}$ : C 63.15, H 5.30, N 20.08; found C 63.29, H 
5.18, N 20.19.

1-甲基-3-甲基-5-(4-三氟甲氧基苯氧基)- $1 H$-吡唑-4甲醛- $O$-[4-(1H-1,2,4-三唑-1-基)苯甲基]肜(8c): 白色固 体, 产率 68\%. m.p. $73 \sim 75{ }^{\circ} \mathrm{C} ;{ }^{1} \mathrm{H}$ NMR $(400 \mathrm{MHz}$, $\left.\mathrm{CDCl}_{3}\right) \delta: 8.58(\mathrm{~s}, 1 \mathrm{H}$, Triazole-H), $8.12(\mathrm{~s}, 1 \mathrm{H}$, Triazole$\mathrm{H}), 7.83(\mathrm{~s}, 1 \mathrm{H}, \mathrm{CH}=\mathrm{N}), 7.63(\mathrm{~d}, J=8.4 \mathrm{~Hz}, 2 \mathrm{H}, \mathrm{ArH})$, 7.41 (d, $J=8.4 \mathrm{~Hz}, 2 \mathrm{H}, \operatorname{ArH}), 7.16$ (d, $J=8.8 \mathrm{~Hz}, 2 \mathrm{H}$, $\operatorname{ArH}), 6.89$ (d, J=8.8 Hz, 2H, ArH), 5.00 (s, 2H, $\left.\mathrm{CH}_{2}\right)$, $3.62\left(\mathrm{~s}, 3 \mathrm{H}, \mathrm{NCH}_{3}\right), 2.35\left(\mathrm{~s}, 3 \mathrm{H}, \mathrm{CH}_{3}\right) ;{ }^{13} \mathrm{C} \mathrm{NMR}(100$ $\left.\mathrm{MHz} \mathrm{CDCl}_{3}\right) \delta: 154.9,147.1,144.8,140.7,138.0,136.5$, 129.7, 129.4, 120.8 (q, $J=176 \mathrm{~Hz}$ ), 116.1, 100.1, 75.1, 34.3, 14.5. Anal. calcd for $\mathrm{C}_{22} \mathrm{H}_{19} \mathrm{~F}_{3} \mathrm{~N}_{6} \mathrm{O}_{3}$ : C 55.93, $\mathrm{H} 4.05$, $\mathrm{N}$ 17.79; found C 55.76, H 4.21, N 17.92.

1-甲基-3-甲基-5-(3-甲基苯氧基)- $1 H$-吡唑-4-甲醛$O$-[4-(1H-1,2,4-三唑-1-基)苯甲基]肟(8d): 白色固体, 产 率 64\%. m.p. 71 $73{ }^{\circ} \mathrm{C} ;{ }^{1} \mathrm{H}$ NMR (400 MHz, $\left.\mathrm{CDCl}_{3}\right) \delta$ : 8.56 (s, 1H, Triazole-H), 8.11 (s, 1H, Triazole-H), 7.83 (s, $1 \mathrm{H}, \mathrm{CH}=\mathrm{N}), 7.61(\mathrm{~d}, J=8.4 \mathrm{~Hz}, 2 \mathrm{H}, \mathrm{ArH}), 7.43(\mathrm{~d}, J=$ $8.4 \mathrm{~Hz}, 2 \mathrm{H}, \mathrm{ArH}), 6.66 \sim 7.20(\mathrm{~m}, 4 \mathrm{H}, \mathrm{ArH}), 5.05$ (s, 2H, $\left.\mathrm{CH}_{2}\right), 3.59$ (s, 3H, $\left.\mathrm{NCH}_{3}\right), 2.37\left(\mathrm{~s}, 3 \mathrm{H}, \mathrm{CH}_{3}\right), 2.31(\mathrm{~s}, 3 \mathrm{H}$, $\left.\mathrm{CH}_{3}\right) ;{ }^{13} \mathrm{C} \mathrm{NMR}\left(100 \mathrm{MHz}, \mathrm{CDCl}_{3}\right) \delta: 156.7,152.6,147.9$, $146.8,141.2,140.8,140.3,138.2,136.4,129.8,129.7$, $124.5,119.9,115.8,112.2,100.2,75.0,34.2,21.4,14.8$. Anal. calcd for $\mathrm{C}_{22} \mathrm{H}_{22} \mathrm{~N}_{6} \mathrm{O}_{2}$ : C 65.66, H 5.51, N 20.88; found C 65.49, H 5.63, N 20.78.

1-甲基-3-甲基-5-(4-甲基苯氧基)- $1 H$-吡唑-4-甲醛$O$-[4-(1H-1,2,4-三唑-1-基)苯甲基]肜(8e)：白色固体，产 率 70\%. m.p. $63 \sim 65{ }^{\circ} \mathrm{C} ;{ }^{1} \mathrm{H}$ NMR $\left(400 \mathrm{MHz}, \mathrm{CDCl}_{3}\right) \delta$ : 8.58 (s, 1H, Triazole-H), 8.12 (s, 1H, Triazole-H), 7.80 (s, $1 \mathrm{H}, \mathrm{CH}=\mathrm{N}), 7.62(\mathrm{~d}, J=8.0 \mathrm{~Hz}, 2 \mathrm{H}, \mathrm{ArH}), 7.43(\mathrm{~d}, J=$ $8.0 \mathrm{~Hz}, 2 \mathrm{H}, \mathrm{ArH}), 7.10$ (d, J=8.4 Hz, 2H, ArH), 6.78 (d, $J=8.4 \mathrm{~Hz}, 2 \mathrm{H}, \mathrm{ArH}), 5.05\left(\mathrm{~s}, 2 \mathrm{H}, \mathrm{CH}_{2}\right), 3.62(\mathrm{~s}, 3 \mathrm{H}$, $\left.\mathrm{N}-\mathrm{CH}_{3}\right), 2.38\left(\mathrm{~s}, 3 \mathrm{H}, \mathrm{CH}_{3}\right), 2.31\left(\mathrm{~s}, 3 \mathrm{H}, \mathrm{CH}_{3}\right) ;{ }^{13} \mathrm{C} \mathrm{NMR}$ $\left(100 \mathrm{MHz}, \mathrm{CDCl}_{3}\right) \delta: 154.7,152.6,148.1,146.8,144.7$, $141.2,138.2,136.4,133.2,130.4,129.8,122.8,119.9$, 116.3, 115.1, 100.1, 75.0, 34.2, 20.6, 14.8. Anal. calcd for $\mathrm{C}_{22} \mathrm{H}_{22} \mathrm{~N}_{6} \mathrm{O}_{2}$ : C 65.66, H 5.51, N 20.88; found C 65.82, H 5.37, N 20.97.

1- 甲基-3-甲基-5-苯氧基- $1 H$-吡唑-4-甲醛- $O$ - [4(1H-1,2,4-三唑-1-基)苯甲基]肟(8f)：白色固体，产率 66\%. m.p. 86 88 ${ }^{\circ} \mathrm{C} ;{ }^{1} \mathrm{H}$ NMR (400 MHz, $\left.\mathrm{CDCl}_{3}\right) \delta$ : 8.60 (s, 1H, Triazole-H), 8.11 (s, 1H, Triazole-H), 7.83 (s, $1 \mathrm{H}, \mathrm{CH}=\mathrm{N}), 7.61(\mathrm{~d}, J=8.4 \mathrm{~Hz}, 2 \mathrm{H}, \mathrm{ArH}), 7.42(\mathrm{~d}, J=$ $8.4 \mathrm{~Hz}, 2 \mathrm{H}, \mathrm{ArH}), 7.29 \sim 7.33$ (m, 2H, ArH), 6.87 7.12 (m, 3H, ArH), 5.03 (s, 2H, $\mathrm{CH}_{2}$ ), 3.60 (s, 3H, N-CH CH $_{3}, 2.36$ $\left(\mathrm{s}, 3 \mathrm{H}, \mathrm{CH}_{3}\right) ;{ }^{13} \mathrm{C} \mathrm{NMR}\left(100 \mathrm{MHz}, \mathrm{CDCl}_{3}\right) \delta: 156.7,152.5$, $147.7,146.9,141.1,140.8,138.1,136.4,130.0,129.8$, $123.7,119.9,115.3,100.2,75.0,34.2,14.7$. Anal. calcd for $\mathrm{C}_{21} \mathrm{H}_{20} \mathrm{~N}_{6} \mathrm{O}_{2}$ : C 64.94, H 5.19, N 21.64; found C 64.79, $\mathrm{H}$ 5.02, N 21.76.

1-甲基-3-甲基-5-(2-氟苯氧基)- $1 H$-吡唑-4-甲醛$O$-[4-(1H-1,2,4-三唑-1-基)苯甲基]肟 $(8 \mathrm{~g})$ ：白色固体，产 率 61\%. m.p. 77 $79{ }^{\circ} \mathrm{C} ;{ }^{1} \mathrm{H}$ NMR $\left(400 \mathrm{MHz}, \mathrm{CDCl}_{3}\right) \delta$ : 8.56 (s, 1H, Triazole-H), 8.11 (s, 1H, Triazole-H), 7.82 (s, $1 \mathrm{H}, \mathrm{CH}=\mathrm{N}), 7.62(\mathrm{~d}, J=8.4 \mathrm{~Hz}, 2 \mathrm{H}, \mathrm{ArH}), 7.41(\mathrm{~d}, J=$ $8.4 \mathrm{~Hz}, 2 \mathrm{H}, \mathrm{ArH}), 6.73 \sim 7.19(\mathrm{~m}, 4 \mathrm{H}, \mathrm{ArH}), 5.01$ (s, 2H, $\left.\mathrm{CH}_{2}\right), 3.65\left(\mathrm{~s}, 3 \mathrm{H}, \mathrm{N}-\mathrm{CH}_{3}\right), 2.34\left(\mathrm{~s}, 3 \mathrm{H}, \mathrm{CH}_{3}\right) ;{ }^{13} \mathrm{C} \mathrm{NMR}$ $\left(100 \mathrm{MHz}, \mathrm{CDCl}_{3}\right) \delta: 152.6,151.9(\mathrm{~d}, J=247 \mathrm{~Hz}), 147.3$, 147.0, $144.2(\mathrm{~d}, J=11 \mathrm{~Hz}), 140.7,138.1,136.4,129.8$, 129.3, 124.6 (d, $J=4 \mathrm{~Hz}), 124.4$ (d, $J=7 \mathrm{~Hz}), 119.9,117.1$ $(\mathrm{d}, J=18 \mathrm{~Hz}), 116.6,99.8,75.0,34.2,14.5$. Anal. calcd for $\mathrm{C}_{21} \mathrm{H}_{19} \mathrm{FN}_{6} \mathrm{O}_{2}$ : C 62.06, H 4.71, N 20.68; found $\mathrm{C} 62.23, \mathrm{H}$ 4.56, N 20.55 .

1-甲基-3-甲基-5-(3-氟苯氧基)- $1 H$-吡唑-4-甲醛$O$-[4-(1H-1,2,4-三唑-1-基)苯甲基]肟 $(\mathbf{8 h})$ : 白色固体，产 率 63\%. m.p. 81 83 ${ }^{\circ} \mathrm{C} ;{ }^{1} \mathrm{H}$ NMR (400 $\left.\mathrm{MHz}, \mathrm{CDCl}_{3}\right) \delta$ : 8.58 (s, 1H, Triazole-H), 8.12 (s, 1H, Triazole-H), 7.84 (s, $1 \mathrm{H}, \mathrm{CH}=\mathrm{N}), 7.62(\mathrm{~d}, J=8.4 \mathrm{~Hz}, 2 \mathrm{H}, \mathrm{ArH}), 7.41(\mathrm{~d}, J=$ $8.4 \mathrm{~Hz}, 2 \mathrm{H}, \mathrm{ArH}), 6.60 \sim 7.28(\mathrm{~m}, 4 \mathrm{H}, \mathrm{ArH}), 5.03$ (s, 2H, $\left.\mathrm{CH}_{2}\right), 3.61\left(\mathrm{~s}, 3 \mathrm{H}, \mathrm{NCH}_{3}\right), 2.35\left(\mathrm{~s}, 3 \mathrm{H}, \mathrm{CH}_{3}\right) ;{ }^{13} \mathrm{C} \mathrm{NMR}$ $\left(100 \mathrm{MHz}, \mathrm{CDCl}_{3}\right) \delta: 163.5(\mathrm{~d}, J=246 \mathrm{~Hz}), 157.5,152.5$, $147.0,140.7,138.1,136.4,130.8,129.7,129.4,119.9$, 110.7 (d, $J=20 \mathrm{~Hz}), 103.5$ (d, $J=26 \mathrm{~Hz}), 75.1,34.2,14.5$. Anal. calcd for $\mathrm{C}_{21} \mathrm{H}_{19} \mathrm{FN}_{6} \mathrm{O}_{2}$ : C 62.06, H 4.71, N 20.68; found $\mathrm{C} 61.92, \mathrm{H} 4.60$, N 20.84.

1-甲基-3-甲基-5-(4-氟苯氧基)- $1 H$-吡唑-4-甲醛$O$-[4-(1H-1,2,4-三唑-1-基)苯甲基]肜(8i)：白色固体，产 率 65\%. m.p. 86 88 ${ }^{\circ} \mathrm{C} ;{ }^{1} \mathrm{H}$ NMR $\left(400 \mathrm{MHz}, \mathrm{CDCl}_{3}\right) \delta$ : 8.56 (s, 1H, Triazole-H), 8.10 (s, 1H, Triazole-H), 7.81 (s, $1 \mathrm{H}, \mathrm{CH}=\mathrm{N}), 7.62(\mathrm{~d}, J=8.4 \mathrm{~Hz}, 2 \mathrm{H}, \mathrm{ArH}), 7.40(\mathrm{~d}, J=$ $8.4 \mathrm{~Hz}, 2 \mathrm{H}, \mathrm{ArH}), 6.82 \sim 7.00(\mathrm{~m}, 4 \mathrm{H}, \mathrm{ArH}), 5.02$ (s, 2H, $\left.\mathrm{CH}_{2}\right), 3.60\left(\mathrm{~s}, 3 \mathrm{H}, \mathrm{N}-\mathrm{CH}_{3}\right), 2.34\left(\mathrm{~s}, 3 \mathrm{H}, \mathrm{CH}_{3}\right) ;{ }^{13} \mathrm{C} \mathrm{NMR}$ $\left(100 \mathrm{MHz}, \mathrm{CDCl}_{3}\right) \delta: 158.7(\mathrm{~d}, J=241 \mathrm{~Hz}), 152.6,147.7$, $147.0,140.9,138.1,136.4,129.7,129.4,119.9,116.5$ (d, $J=22 \mathrm{~Hz}$ ), 99.9, 75.0, 34.2, 14.6. Anal. calcd for $\mathrm{C}_{21} \mathrm{H}_{19} \mathrm{~F}-$ $\mathrm{N}_{6} \mathrm{O}_{2}$ : C 62.06, H 4.71, N 20.68; found C 62.19, H 4.88, N 20.53 .

1-甲基-3-甲基-5-(4-氯苯氧基)- $1 H$-吡唑-4-甲醛$O$-[4-(1H-1,2,4-三唑-1-基)苯甲基]肜 $(8 \mathbf{j})$ : 白色固体，产 率 71\%. m.p. 93 95 ${ }^{\circ} \mathrm{C} ;{ }^{1} \mathrm{H}$ NMR (400 MHz, $\left.\mathrm{CDCl}_{3}\right) \delta$ : 
8.57 (s, 1H, Triazole-H), 8.11 (s, 1H, Triazole-H), 7.82 (s, $1 \mathrm{H}, \mathrm{CH}=\mathrm{N}), 7.62(\mathrm{~d}, J=8.4 \mathrm{~Hz}, 2 \mathrm{H}, \mathrm{ArH}), 7.38(\mathrm{~d}, J=$ $8.4 \mathrm{~Hz}, 2 \mathrm{H}, \operatorname{ArH}), 7.25(\mathrm{~d}, J=8.8 \mathrm{~Hz}, 2 \mathrm{H}, \operatorname{ArH}), 6.81$ (d, $J=9.2 \mathrm{~Hz}, 2 \mathrm{H}, \mathrm{ArH}), 5.01\left(\mathrm{~s}, 2 \mathrm{H}, \mathrm{CH}_{2}\right), 3.60(\mathrm{~s}, 3 \mathrm{H}$, $\left.\mathrm{NCH}_{3}\right), 2.34$ (s, $\left.3 \mathrm{H}, \mathrm{CH}_{3}\right) ;{ }^{13} \mathrm{C}$ NMR $\left(100 \mathrm{MHz}, \mathrm{CDCl}_{3}\right) \delta$ : $155.2,147.1,141.5,140.8,138.1,137.4,130.0,129.9$, 129.8, 128.7, 119.9, 116.6, 116.4, 100.1, 75.1, 34.2, 14.4. Anal. calcd for $\mathrm{C}_{21} \mathrm{H}_{19} \mathrm{ClN}_{6} \mathrm{O}_{2}$ : C 59.65, H 4.53, N 19.87; found C 59.50, H 4.66, N 19.71.

1- 甲基-3-甲基-5-(2-澳苯氧基)- $1 \mathrm{H}$-吡唑-4-甲醛$O$-[4-(1H-1,2,4-三唑-1-基)苯甲基]肜(8k): 白色固体, 产 率 60\%. m.p. $63 \sim 65{ }^{\circ} \mathrm{C} ;{ }^{1} \mathrm{H}$ NMR $\left(400 \mathrm{MHz}, \mathrm{CDCl}_{3}\right) \delta$ : $8.58(\mathrm{~s}, 1 \mathrm{H}$, Triazole- $\mathrm{H}), 8.12(\mathrm{~s}, 1 \mathrm{H}$, Triazole-H), $7.81(\mathrm{~s}$, $1 \mathrm{H}, \mathrm{CH}=\mathrm{N}), 7.59 \sim 7.62(\mathrm{~m}, 3 \mathrm{H}, \operatorname{ArH}), 7.40(\mathrm{~d}, J=8.0$ $\mathrm{Hz}, 2 \mathrm{H}, \mathrm{ArH}$ ), $6.96 \sim 7.21$ (m, 2H, ArH), 6.64 (d, $J=8.0$ $\mathrm{Hz}, 1 \mathrm{H}, \mathrm{ArH}), 5.01\left(\mathrm{~s}, 2 \mathrm{H}, \mathrm{CH}_{2}\right), 3.63\left(\mathrm{~s}, 3 \mathrm{H}, \mathrm{N}-\mathrm{CH}_{3}\right), 2.35$ (s, $\left.3 \mathrm{H}, \mathrm{CH}_{3}\right) ;{ }^{13} \mathrm{C}$ NMR $\left(100 \mathrm{MHz}, \mathrm{CDCl}_{3}\right) \delta: 153.1,147.1$, 147.0, 140.6, 138.2, 137.2, 136.6, 134.0, 129.8, 128.7, 124.9, 124.4, 120.0, 115.3, 111.4, 100.1, 75.0, 34.3, 14.5. Anal. calcd for $\mathrm{C}_{21} \mathrm{H}_{19} \mathrm{BrN}_{6} \mathrm{O}_{2}$ : C 53.97, H 4.10, N 17.98; found C 54.14, H 4.01, N 17.82.

1-甲基-3-甲基-5-(3-溴苯氧基)- $1 H$-吡唑-4-甲醛$O$-[4-(1H-1,2,4-三唑-1-基)苯甲基]肜(81): 白色固体, 产 率 62\%. m.p. $76 \sim 78{ }^{\circ} \mathrm{C} ;{ }^{1} \mathrm{H}$ NMR $\left(400 \mathrm{MHz}, \mathrm{CDCl}_{3}\right) \delta$ : 8.57 (s, 1H, Triazole-H), 8.11 (s, 1H, Triazole-H), 7.84 (s, $1 \mathrm{H}, \mathrm{CH}=\mathrm{N}), 7.62(\mathrm{~d}, J=8.4 \mathrm{~Hz}, 2 \mathrm{H}, \operatorname{ArH}), 7.39(\mathrm{~d}, J=$ $8.4 \mathrm{~Hz}, 2 \mathrm{H}, \mathrm{ArH}), 6.80 \sim 7.23$ (m, 4H, ArH), 5.01 (s, $2 \mathrm{H}$, $\mathrm{CH}_{2}$ ), 3.60 (s, 3H, $\mathrm{NCH}_{3}$ ), 2.35 (s, 3H, $\left.\mathrm{CH}_{3}\right) ;{ }^{13} \mathrm{C} \mathrm{NMR}$ $\left(100 \mathrm{MHz}, \mathrm{CDCl}_{3}\right) \delta: 157.1,147.1,146.7,140.7,138.1$, $136.4,131.1,129.7,129.5,126.9,123.2,119.9,118.7$, 114.0, 100.3, 75.1, 34.3, 14.4. Anal. calcd for $\mathrm{C}_{21} \mathrm{H}_{19} \mathrm{BrN}_{6}-$ $\mathrm{O}_{2}$ : C 53.97, H 4.10, N 17.98; found C 53.85, H 4.26, N 17.87 .

1-甲基-3-甲基-5-(4-溴苯氧基)- $1 H$-吡唑-4-甲醛$O$-[4-(1H-1,2,4-三唑-1-基)苯甲基]肜(8m): 白色固体, 产 率 69\%. m.p. 101 103 ${ }^{\circ} \mathrm{C} ;{ }^{1} \mathrm{H}$ NMR $\left(400 \mathrm{MHz}, \mathrm{CDCl}_{3}\right)$ $\delta: 8.59(\mathrm{~s}, 1 \mathrm{H}$, Triazole-H), $8.12(\mathrm{~s}, 1 \mathrm{H}$, Triazole-H), 7.82 $(\mathrm{s}, 1 \mathrm{H}, \mathrm{CH}=\mathrm{N}), 7.63(\mathrm{~d}, J=8.4 \mathrm{~Hz}, 2 \mathrm{H}, \mathrm{ArH}), 7.37 \sim 7.41$ (m, 4H, ArH), 6.76 (d, J=9.2 Hz, 2H, ArH), 5.01 (s, $2 \mathrm{H}$, $\mathrm{CH}_{2}$ ), 3.60 (s, 3H, $\mathrm{NCH}_{3}$ ), 2.34 (s, $\left.3 \mathrm{H}, \mathrm{CH}_{3}\right) ;{ }^{13} \mathrm{C} \mathrm{NMR}$ $\left(100 \mathrm{MHz}, \mathrm{CDCl}_{3}\right) \delta: 155.7,147.1,147.0,141.5,140.7$, $138.1,137.3,136.5,132.9,132.8,132.7,129.8,119.9$, 117.0, 116.1, 100.1, 75.1, 34.2, 14.4. Anal. calcd for $\mathrm{C}_{21} \mathrm{H}_{19} \mathrm{BrN}_{6} \mathrm{O}_{2}$ : C 53.97, H 4.10, N 17.98; found C 53.90, H 3.96, N 18.10 .
1-甲基-3-甲基-5-(2,3-二氟苯氧基)- $1 H$-吡唑-4-甲醛$O$-[4-(1H-1,2,4-三唑-1-基)苯甲基]肜(8n): 白色固体, 产 率 60\%. m.p. 66 68 ${ }^{\circ} \mathrm{C} ;{ }^{1} \mathrm{H}$ NMR $\left(400 \mathrm{MHz}, \mathrm{CDCl}_{3}\right) \delta$ : 8.58 (s, 1H, Triazole-H), 8.12 (s, 1H, Triazole-H), 7.84 (s, $1 \mathrm{H}, \mathrm{CH}=\mathrm{N}), 7.62(\mathrm{~d}, J=8.4 \mathrm{~Hz}, 2 \mathrm{H}, \mathrm{ArH}), 7.40(\mathrm{~d}, J=$ $8.4 \mathrm{~Hz}, 2 \mathrm{H}, \mathrm{ArH}), 6.86 \sim 6.94(\mathrm{~m}, 2 \mathrm{H}, \mathrm{ArH}), 6.48 \sim 6.52$ (m, $1 \mathrm{H}, \mathrm{ArH}), 5.00\left(\mathrm{~s}, 2 \mathrm{H}, \mathrm{CH}_{2}\right), 3.66\left(\mathrm{~s}, 3 \mathrm{H}, \mathrm{NCH}_{3}\right), 2.33$ (s, $\left.3 \mathrm{H}, \mathrm{CH}_{3}\right) ;{ }^{13} \mathrm{C}$ NMR $\left(100 \mathrm{MHz}, \mathrm{CDCl}_{3}\right) \delta: 152.5,151.5$ (d, $J=248 \mathrm{~Hz}$ ), 150.3, 147.1, 146.6, 145.6, 142.3, 140.8, 140.4, 138.1, 136.4, 129.6, 123.4, 119.9, 112.2 (d, $J=17$ $\mathrm{Hz}), 111.4,99.9,75.1,34.3,14.2$. Anal. calcd for $\mathrm{C}_{21} \mathrm{H}_{18} \mathrm{~F}_{2^{-}}$ $\mathrm{N}_{6} \mathrm{O}_{2}:$ C 59.43, H 4.27, N 19.80; found C 59.61, H 4.13, N 19.96.

1-甲基-3-甲基-5-(2,4-二氟苯氧基)- $1 H$-吡唑-4-甲醛$O$-[4-(1H-1,2,4-三唑-1-基)苯甲基]肜(8o)：白色固体，产 率 62\%. m.p. $126 \sim 128{ }^{\circ} \mathrm{C}$; ${ }^{1} \mathrm{H}$ NMR $\left(400 \mathrm{MHz}, \mathrm{CDCl}_{3}\right)$ $\delta: 8.58(\mathrm{~s}, 1 \mathrm{H}$, Triazole-H), $8.12(\mathrm{~s}, 1 \mathrm{H}$, Triazole-H), 7.82 $(\mathrm{s}, 1 \mathrm{H}, \mathrm{CH}=\mathrm{N}), 7.63(\mathrm{~d}, J=8.4 \mathrm{~Hz}, 2 \mathrm{H}, \mathrm{ArH}), 7.41(\mathrm{~d}$, $J=8.4 \mathrm{~Hz}, 2 \mathrm{H}, \mathrm{ArH}), 6.72 \sim 6.94(\mathrm{~m}, 3 \mathrm{H}, \mathrm{ArH}), 5.01$ (s, $\left.2 \mathrm{H}, \mathrm{CH}_{2}\right), 3.66\left(\mathrm{~s}, 3 \mathrm{H}, \mathrm{NCH}_{3}\right), 2.32\left(\mathrm{~s}, 3 \mathrm{H}, \mathrm{CH}_{3}\right) .{ }^{13} \mathrm{C}$ NMR (100 MHz, $\left.\mathrm{CDCl}_{3}\right) \delta: 158.7$ (d, $\left.J=244 \mathrm{~Hz}\right), 152.5$, $150.5,147.1,140.8,140.5,138.1,136.4,129.6,124.5$, $119.9,117.3$ (d, $J=9 \mathrm{~Hz}), 115.8,112.3,111.1,105.6,99.6$, 75.0, 34.2, 14.3. Anal. calcd for $\mathrm{C}_{21} \mathrm{H}_{18} \mathrm{~F}_{2} \mathrm{~N}_{6} \mathrm{O}_{2}$ : C 59.43, $\mathrm{H}$ 4.27, N 19.80; found C 59.30, H 4.43, N 19.63.

1-甲基-3-甲基-5-(3,5-二氟苯氧基)- $1 H$-吡唑-4-甲醛$O$-[4-(1H-1,2,4-三唑-1-基)苯甲基]肜(8p)：白色固体，产 率 64\%. m.p. 89 91 ${ }^{\circ} \mathrm{C} ;{ }^{1} \mathrm{H}$ NMR $\left(400 \mathrm{MHz}, \mathrm{CDCl}_{3}\right) \delta$ : $8.56(\mathrm{~s}, 1 \mathrm{H}$, Triazole-H), 8.12 (s, 1H, Triazole-H), 7.85 (s, $1 \mathrm{H}, \mathrm{CH}=\mathrm{N}), 7.62(\mathrm{~d}, J=8.4 \mathrm{~Hz}, 2 \mathrm{H}, \mathrm{ArH}), 7.40(\mathrm{~d}, J=$ $8.4 \mathrm{~Hz}, 2 \mathrm{H}, \mathrm{ArH}), 6.40 \sim 6.56$ (m, $3 \mathrm{H}, \mathrm{ArH}), 5.02(\mathrm{~s}, 2 \mathrm{H}$, $\mathrm{CH}_{2}$ ), 3.61 (s, 3H, $\mathrm{NCH}_{3}$ ), 2.35 (s, $\left.3 \mathrm{H}, \mathrm{CH}_{3}\right) ;{ }^{13} \mathrm{C} \mathrm{NMR}$ $\left(100 \mathrm{MHz}, \mathrm{CDCl}_{3}\right) \delta: 163.7$ (d, $\left.J=248 \mathrm{~Hz}\right), 158.1,157.9$, $152.6,147.2,146.1,140.8,140.5,138.1,136.4,129.6$, 119.9, 100.4, 99.4 (d, $J=30 \mathrm{~Hz}), 75.1,34.3$, 14.3. Anal. calcd for $\mathrm{C}_{21} \mathrm{H}_{18} \mathrm{~F}_{2} \mathrm{~N}_{6} \mathrm{O}_{2}$ : C 59.43, H 4.27, N 19.80; found C 59.25, H 4.39, N 19.91 .

1-甲基-3-甲基-5-(2,4-二氯苯氧基)- $1 H$-吡唑-4-甲醛$O$-[4-(1H-1,2,4-三唑-1-基)苯甲基]肜(8q)：白色固体，产 率 63\%. m.p. $103 \sim 105{ }^{\circ} \mathrm{C} ;{ }^{1} \mathrm{H}$ NMR $\left(400 \mathrm{MHz}, \mathrm{CDCl}_{3}\right)$ $\delta: 8.57$ (s, 1H, Triazole-H), 8.11 (s, 1H, Triazole-H), 7.82 $(\mathrm{s}, 1 \mathrm{H}, \mathrm{CH}=\mathrm{N}), 7.62(\mathrm{~d}, J=8.4 \mathrm{~Hz}, 2 \mathrm{H}, \mathrm{ArH}), 7.36 \sim 7.42$ (m, 3H, ArH), 7.06 7.09 (m, 1H, ArH), 6.58 (d, $J=8.8$ $\mathrm{Hz}, 1 \mathrm{H}, \mathrm{ArH}), 4.99$ (s, 2H, CH 2$), 3.63$ (s, 3H, $\left.\mathrm{NCH}_{3}\right), 2.33$ (s, $\left.3 \mathrm{H}, \mathrm{CH}_{3}\right) ;{ }^{13} \mathrm{C} \mathrm{NMR}\left(100 \mathrm{MHz}, \mathrm{CDCl}_{3}\right) \delta: 152.6,150.8$, 
$147.2,146.5,140.4,136.9,136.4,130.5,129.6,129.4$, 129.0, 127.9, 123.6, 119.8, 116.1, 100.0, 75.1, 34.2, 14.1. Anal. calcd for $\mathrm{C}_{21} \mathrm{H}_{18} \mathrm{Cl}_{2} \mathrm{~N}_{6} \mathrm{O}_{2}$ : C 55.15, H 3.97, $\mathrm{N}$ 18.38; found C 55.31, H 3.88, N 18.25.

1-(4-甲基苯基)-3-甲基-5-(4-甲基苯氧基)- $1 H$-吡唑4-甲醛- $O$-[4-(1H-1,2,4-三唑-1-基)苯甲基]肜(8r): 白色 固体, 产率 66\%. m.p. 91 93 ${ }^{\circ} \mathrm{C} ;{ }^{1} \mathrm{H}$ NMR (400 MHz, $\left.\mathrm{CDCl}_{3}\right) \delta: 8.57(\mathrm{~s}, 1 \mathrm{H}$, Triazole-H), $8.12(\mathrm{~s}, 1 \mathrm{H}$, Triazole$\mathrm{H}), 7.85(\mathrm{~s}, 1 \mathrm{H}, \mathrm{CH}=\mathrm{N}), 7.63(\mathrm{~d}, J=8.0 \mathrm{~Hz}, 2 \mathrm{H}, \mathrm{ArH})$, 7.45 (d, $J=8.0 \mathrm{~Hz}, 4 \mathrm{H}, \operatorname{ArH}), 7.14$ (d, $J=8.0 \mathrm{~Hz}, 2 \mathrm{H}$, ArH), 7.04 (d, $J=8.4 \mathrm{~Hz}, 2 \mathrm{H}, \operatorname{ArH}), 6.78$ (d, $J=8.8 \mathrm{~Hz}$, $2 \mathrm{H}, \mathrm{ArH}), 5.07\left(\mathrm{~s}, 2 \mathrm{H}, \mathrm{CH}_{2}\right), 2.44\left(\mathrm{~s}, 3 \mathrm{H}, \mathrm{CH}_{3}\right), 2.32(\mathrm{~s}$, $\left.3 \mathrm{H}, \mathrm{CH}_{3}\right), 2.27\left(\mathrm{~s}, 3 \mathrm{H}, \mathrm{CH}_{3}\right) ;{ }^{13} \mathrm{C}$ NMR $\left(100 \mathrm{MHz}, \mathrm{CDCl}_{3}\right)$ $\delta: 154.8,148.1,147.5,141.4,141.1,138.2,137.1,135.1$, $133.1,130.4,130.2,130.0,129.9,129.7,122.1,120.0$, 115.3, 101.6, 75.1, 21.0, 20.6, 15.1. Anal. calcd for $\mathrm{C}_{28} \mathrm{H}_{26} \mathrm{~N}_{6} \mathrm{O}_{2}$ : C 70.28, H 5.48, N 17.56; found C 70.10, H $5.60, \mathrm{~N} 17.41$.

\section{6 生物活性测试}

\subsection{1 杀虫试验方法}

用分析天平称取一定质量的原药, 用含吐温-80 乳 化剂的 DMF 溶解配制成 $1.0 \%$ 母液, 然后用蒸馏水稀释 备用. 每个处理 3 次重复, 设空白对照. 杀虫活性测试 所选昆虫分别为朱砂叶螨(Tetranychus cinnabarinus)、蚜 虫(Aphis medicaginis)和粘虫(Oriental armyworm). 对照 药剂分别为唑螨酯(Fenpyroximate)、吡虫啉(Imidacloprid)和阿维菌素(Avermectin).

朱砂叶螨和蚜虫: 采用喷雾法. 首先, 分别将接有 朱砂叶螨和蚜虫的蚕豆叶片于 Potter 喷雾塔下喷雾处 理, 处理后朱砂叶螨置于 $24 \sim 27{ }^{\circ} \mathrm{C}$ 观察室内培养, 蚜 虫置于 $20 \sim 22{ }^{\circ} \mathrm{C}$ 观察室内培养, 调查药后 $2 \mathrm{~d}$ 的死活 虫数, 并进行统计分析.

粘虫: 采用浸叶碟法. 首先, 将适量玉米叶在配好 的药液中充分浸润后自然阴干, 放入垫有滤纸的培养血 中, 接粘虫 3 龄中期幼虫 10 头/皿, 置于 $24 \sim 27{ }^{\circ} \mathrm{C}$ 观察 室内培养, 调查药后 $2 \mathrm{~d}$ 的死活虫数, 并进行统计分析.

\section{6 .2 抗肿瘤活性测试}

所用细胞株分别为人肝癌(SMMC-7721)和人胰腺 癌(Panc-1)细胞株. 阳性对照药剂分别为 5-氟尿嘧啶 (5-Fluorouracil)和索拉菲尼(Sorafenib). 采用 MTT 法测 定了目标化合物的体外抗肿瘤活性, 具体方法参照文献 [31].

辅助材料(Supporting Information) 化合物 8a $\sim 8 r$ 的 ${ }^{1} \mathrm{H}$ NMR 和 ${ }^{13} \mathrm{C}$ NMR 图谱. 这些材料可以免费从本刊网 站(http://sioc-journal.cn/)上下载.

\section{References}

[1] Liu, X. H.; Zhao, W.; Shen, Z. H.; Xing, J. H.; Yuan, J.; Yang, G.; Xu, T. M.; Peng, W. L. Bioorg. Med. Chem. Lett. 2016, 26, 3626.

[2] Zhai, Z. W.; Wang, Q.; Shen, Z. H.; Tan, C. X.; Weng, J. Q.; Liu, X H. Chin. J. Org. Chem. 2017, 37, 232 (in Chinese). (翟志文，汪乔，沈钟华，谭成侠，翁建全，刘幸海，有机化学， 2017, 37, 232.)

[3] Liu, X. H.; Zhao, W.; Shen, Z. H.; Xing, J. H.; Xu, T. M.; Peng, W. L. Eur. J. Med. Chem. 2017, 125, 881.

[4] Liu, X. H.; Wang, Q.; Sun, Z. H.; Wedge, D. E.; Becnel, J. J.; Estep, A. S.; Tan, C. X. Weng, J. Q. Pest Manage. Sci. 2017, 73, 953.

[5] Shi, J. J.; Ren, G. H.; Wu, N. J.; Weng, J. Q.; Xu, T. M.; Liu, X. H.; Tan, C. X. Chin. Chem. Lett. 2017, 28, 1727.

[6] Li, Q. M.; Pang, K. S.; Zhao, J. P.; Liu, X. H.; Weng, J. Q. Chin. J. Org. Chem. 2017, 37, 1009 (in Chinese). (李倩梅, 庞凯胜, 赵建平, 刘幸海, 翁建全, 有机化学, 2017, 37, 1009.)

[7] Dai, X. Q.; Zhu, Y. B.; Wang, Z. Y.; Weng, J. Q. Chin. J. Org. Chem. 2017, 37, 1924 (in Chinese) (戴小强, 朱亚波, 汪洲洋, 翁建全, 有机化学, 2017, 37, 1924.)

[8] Shi, J. J.; Ren, G. H.; Dai, Z. M.; Wu, N, J.; Weng, J. Q.; Xu, T. M.; Liu, X. H.; Tan, C. X. Lett. Drug Des. Discovery 2018, 15, 15.

[9] Shen, Z. H.; Sun, Z. H.; Becnel, J. J.; Estep, A.; Wedge, D. E.; Tan, C. X.; Weng, J. Q.; Han, L.; Liu, X. H. Lett. Drug Des. Discovery 2018, 15,951 .

[10] Chen, L.; Shen, Z. H.; Xu, T. M.; Tan, C. X.; Weng, J. Q.; Han, L.; Peng. W. L.; Liu, X. H. J. Heterocycl. Chem. 2018, 55, 946.

[11] Li, Y.; Zhang, H. Q.; Liu, J.; Yang, X. P.; Liu, Z. J. J. Agric. Food Chem. 2006, 54, 3636.

[12] Ouyang, G. P.; Cai, X. J.; Chen, Z.; Song, B. A.; Bhadury, P. S.; Yang, S.; Jin, L. H.; Xue, W.; Hu, D. Y.; Zeng, S. J. Agric. Food Chem. 8, 56, 10160.

[13] Wang, S. L.; Shi, Y. J.; He, H. B.; Li, Y.; Li, Y.; Dai, H. Chin. Chem. Lett. 2015, 26, 672

[14] Dai, H.; Ge, S. S.; Guo, J.; Chen, S.; Huang, M. L.; Yang. J. Y.; Sun, S. Y.; Ling, Y.; Shi, Y. J. Eur. J. Med. Chem. 2018, 143, 1066.

[15] Hamaguchi, H.; Kajihara, O.; Katoh, M. J. Pestic. Sci. 1995, 20, 173.

[16] Fu, C. R.; Peng, J.; Ning, Y.; Liu, M.; Shan, P. C.; Liu, J.; Li, Y. Q.; Hu, F. Z.; Zhu, Y. Q.; Yang, H. Z.; Zou, X. M. Pest Manage. Sci. 2014, 70, 1207.

[17] Dai, H.; Xiao, Y. S.; Li, Z.; Xu, X. Y.; Qian, X. H. Chin. Chem. Lett. 2014, 25, 1014.

[18] Zhou, Q.; Zheng, D. D.; Shi, Y. J.; Yao, W.; Qian, H. W.; Ding, Y.; Wei, Z. H.; Shen, A. Y.; Feng, X.; Shi, J.; Dai, H. Chin. J. Org. Chem. 2018, 38, 3318 (in Chinese) (周钱, 郑丹丹, 石玉军, 姚炜, 钱宏炜, 丁颖, 魏中吴, 沈爱宝, 冯霞, 石健, 戴红, 有机化学, 2018, 38, 3318.)

[19] Lü, X. H.; Li, Q. S.; Ren, Z. L.; Chu, M. J.; Sun, J.; Zhang, X.; Xing, M.; Zhu, H. L.; Cao, H. Q. Eur. J. Med. Chem. 2015, 108, 586.

[20] Dai, H.; Ge, S. S.; Li, G.; Chen, J.; Shi, Y. J.; Ye, L. Y.; Ling, Y. Bioorg. Med. Chem. Lett. 2016, 26, 4504.

[21] Clark, R. L.; Clements, C. J.; Barrett, M. P.; Mackay, S. P.; Rathnam, R. P.; Owusu-Dapaah, G.; Spencer, J.; Huggan, J. K. Bioorg. Med. Chem. Lett. 2012, 20, 6019.

[22] Jia, C. Q.; Su, W. C.; Xu, Y. J.; Liu, J. P.; Qin, Z. H. Chin. J. Org. Chem. 2016, 36, 830 (in Chinese).

(贾长青，苏旺苍，徐彦军，刘吉平，覃兆海，有机化学，2016, 36, 830.)

[23] Wang, H. L.; Ruan, L. L.; Chen, Y.; Liu, X. H.; Weng, J. Q. Chin. J. Org. Chem. 2014, 34, 419 (in Chinese).

(王海林, 阮铃莉, 陈勇, 刘幸海, 翁建全, 有机化学, 2014, 34, 419.)

[24] El-Helby, A. G. A. Bull. Pharm. Sci. 2005, 28, 45. 
[25] El-Sherief, H. A. M.; Youssif, B. G.; Bukhari, S. N. A.; Abdelazeem, A. H.; Abdel-Aziz, M.; Abdel-Rahman, H. M. Eur. J. Med. Chem. 2018, 156, 774.

[26] Jia, C. Q.; Yang, D. Y.; Che, C. L.; Ma, Y. Q.; Rui, C. H.; Yan, X. J.; Qin, Z. H. Chem. J. Chin. Univ. 2016, 37, 892 (in Chinese). (贾长青, 杨冬燕, 车传亮, 马永强, 茌昌辉, 间晓静, 覃兆海, 高等学校化学学报, 2016, 37, 892.)

[27] Wang, H. L.; Ruan, L. L.; Chen, Y.; Liu, X. H.; Weng, J. Q. Chin. J. Org. Chem. 2018, 38, 2137 (in Chinese)

(魏光璞, 张茜, 雷强, 徐沝, 张明千, 龙跃, 有机化学, 2018, 38, 2137.)
[28] Park, M. S.; Park, H. J.; Park, K. H.; Lee, K. I. Synth. Commun. 2004, 34, 1541.

[29] Park, H. J.; Lee, K.; Park, S. J.; Ahn, B.; Lee, J. C.; Cho, H. Y.; Lee, K. I. Bioorg. Med. Chem. Lett. 2005, 15, 3307.

[30] Tanaka, A.; Terasawa, T.; Hagihara, H.; Sakuma, Y.; Ishibe, N.; Sawada, M.; Takasugi, H.; Tanaka, H. J. Med. Chem. 1998, 41, 2390.

[31] Liu, J. C.; Liu, Y. J.; He, H. W. Chin. J. Org. Chem. 2015, 35, 462 (in Chinese).

(刘建超, 刘勇军, 贺红武, 有机化学, 2015, 35, 462.)

(Li, L.; Fan, Y.) 